\title{
Crystal Phase Quantum Well Emission with Digital Control
}

Assali, S.; Laehnemann, J.; Vu, Thi Thu Trang; Jöns, K. D.; Gagliano, L.; Verheijen, M. A.; Akopian, Nika; Bakkers, E. P. A. M.; Haverkort, J. E. M.

Published in:

Nano Letters

Link to article, DOI:

10.1021/acs.nanolett.7b02489

Publication date:

2017

Document Version

Publisher's PDF, also known as Version of record

Link back to DTU Orbit

Citation (APA):

Assali, S., Laehnemann, J., Vu, T. T. T., Jöns, K. D., Gagliano, L., Verheijen, M. A., Akopian, N., Bakkers, E. P. A. M., \& Haverkort, J. E. M. (2017). Crystal Phase Quantum Well Emission with Digital Control. Nano Letters, 17(10), 6062-6068. https://doi.org/10.1021/acs.nanolett.7b02489

\section{General rights}

Copyright and moral rights for the publications made accessible in the public portal are retained by the authors and/or other copyright owners and it is a condition of accessing publications that users recognise and abide by the legal requirements associated with these rights.

- Users may download and print one copy of any publication from the public portal for the purpose of private study or research.

- You may not further distribute the material or use it for any profit-making activity or commercial gain

- You may freely distribute the URL identifying the publication in the public portal 


\title{
Crystal Phase Quantum Well Emission with Digital Control
}

\author{
S. Assali, ${ }^{\dagger, \perp}$ J. Lähnemann, ${ }^{\ddagger \odot}$ T. T. T. Vu, ${ }^{\dagger}$ K. D. Jöns, ${ }^{\S, \# \odot ~ L . ~ G a g l i a n o, ~}{ }^{\dagger \odot}$ M. A. Verheijen, ${ }^{\dagger, \|}$ \\ N. Akopian, ${ }^{\dagger, \S, \nabla}$ E. P. A. M. Bakkers, ${ }^{\dagger, \S(0)}$ and J. E. M. Haverkort ${ }^{*}{ }^{\dagger}$ \\ ${ }^{\dagger}$ Department of Applied Physics, Eindhoven University of Technology, 5600 MB, Eindhoven, The Netherlands \\ ${ }^{\ddagger}$ Paul-Drude-Institut für Festkörperelektronik, Hausvogteiplatz 5-7, 10117 Berlin, Germany \\ ${ }^{\S}$ Kavli Institute of Nanoscience, Delft University of Technology, 2600 GA, Delft, The Netherlands \\ "Philips Innovation Services Eindhoven, High Tech Campus 11, 5656 AE, Eindhoven, The Netherlands
}

Supporting Information

ABSTRACT: One of the major challenges in the growth of quantum well and quantum dot heterostructures is the realization of atomically sharp interfaces. Nanowires provide a new opportunity to engineer the band structure as they facilitate the controlled switching of the crystal structure between the zinc-blende (ZB) and wurtzite (WZ) phases. Such a crystal phase switching results in the formation of crystal phase quantum wells (CPQWs) and quantum dots (CPQDs). For GaP CPQWs, the inherent electric fields due to the discontinuity of the spontaneous polarization at the WZ/ZB
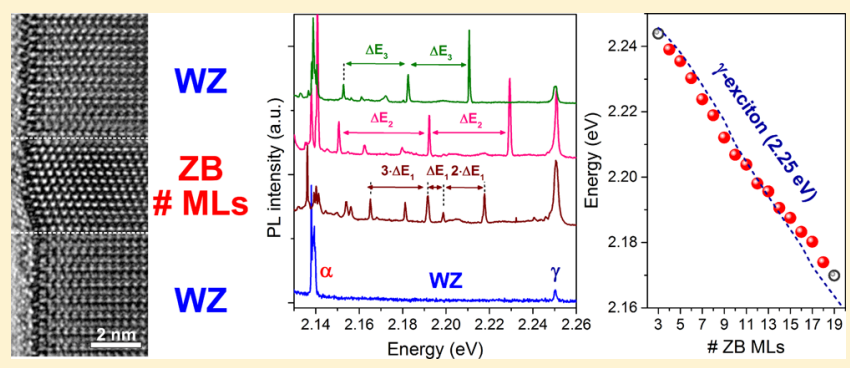
junctions lead to the confinement of both types of charge carriers at the opposite interfaces of the WZ/ZB/WZ structure. This confinement leads to a novel type of transition across a ZB flat plate barrier. Here, we show digital tuning of the visible emission of $\mathrm{WZ} / \mathrm{ZB} / \mathrm{WZ} \mathrm{CPQWs}$ in a $\mathrm{GaP}$ nanowire by changing the thickness of the $\mathrm{ZB}$ barrier. The energy spacing between the sharp emission lines is uniform and is defined by the addition of single $\mathrm{ZB}$ monolayers. The controlled growth of identical quantum wells with atomically flat interfaces at predefined positions featuring digitally tunable discrete emission energies may provide a new route to further advance entangled photons in solid state quantum systems.

KEYWORDS: Semiconductor nanowire, gallium phosphide, crystal phase quantum well, spontaneous polarization, photoluminescence

uantum confinement of electrons and holes in semiconductors is commonly realized by combining semiconductors with different bandgaps in quantum well $(\mathrm{QW})$ and quantum dot (QD) heterostructures. However, alloy fluctuations and interface roughness prevent a precise control over the emission energy. Consequently, fine-tuning of each individual QD emission energy is required by using the Stark effect or strain methods, ${ }^{1-4}$ which severely limits the use of heterostructures as identical single-photon emission sources in photonic circuits. ${ }^{5}$ To overcome these limitations, homojunctions made of zinc blende (ZB) and wurtzite (WZ) crystal phases can be grown with a high degree of control in semiconductor nanowires (NWs). ${ }^{6-9}$ This method allows the fabrication of atomically sharp crystal phase quantum well (CPQW) and quantum dot (CPQD) structures with monolayer thickness control, allowing digitally tuning of the emission wavelength. ${ }^{10}$ In this type of homojunctions, the band offset between the $\mathrm{WZ}$ and $\mathrm{ZB}$ phases results in carrier confinement within the $\mathrm{WZ}$ phase at either side of the ZB barrier ${ }^{7,11-14}$ while avoiding residual strain and alloy intermixing as in the case of compositional heterojunctions. We will refer to this novel charge confinement structure as a CPQW. Due to the atomically flat confinement potentials in these CPQWs, the line width of the QW emission can be significantly reduced as compared to a compositional QW. Optical emission from confined states in CPQWs with different charge confine- ment geometries has been shown in InP and GaAs nanowires, ${ }^{8,10,11}$ where the emission of single-photons and cascaded photon-pairs was demonstrated. ${ }^{15}$

Recently, highly reproducible crystal-phase switching was shown in GaP nanowires, with a control over the $\mathrm{ZB}$ segment length approaching the monolayer $(\mathrm{ML})$ level. ${ }^{6}$ In this material system, the $\mathrm{ZB}$ phase has an indirect band gap, ${ }^{16,17}$ while a quasidirect band gap is observed in the $\mathrm{WZ}$ phase, promising a route to single-photon emission in the visible spectral range. ${ }^{18-20}$ In addition, strong spontaneous polarization fields across the WZ/ $\mathrm{ZB} / \mathrm{WZ}$ segments are predicted for $\mathrm{GaP} .^{21}$ The discontinuity of the spontaneous polarization $P_{S P}$ induces polarization sheet charges at the $\mathrm{WZ} / \mathrm{ZB} / \mathrm{WZ}$ interfaces, ${ }^{22}$ resulting in the quantum-confined Stark effect (QCSE) where the carrier confinement is determined by the polarization-induced triangular potentials. $^{23,24}$ Since the ZB barrier is atomically flat, the separation between the positively and the negatively charged carrier sheets is precisely defined, thus avoiding any broadening effects due to spatial inhomogeneities in the electrostatic and confinement potentials. This behavior has been investigated in

Received: June 13, 2017

Revised: September 6, 2017

Published: September 11, 2017 
a

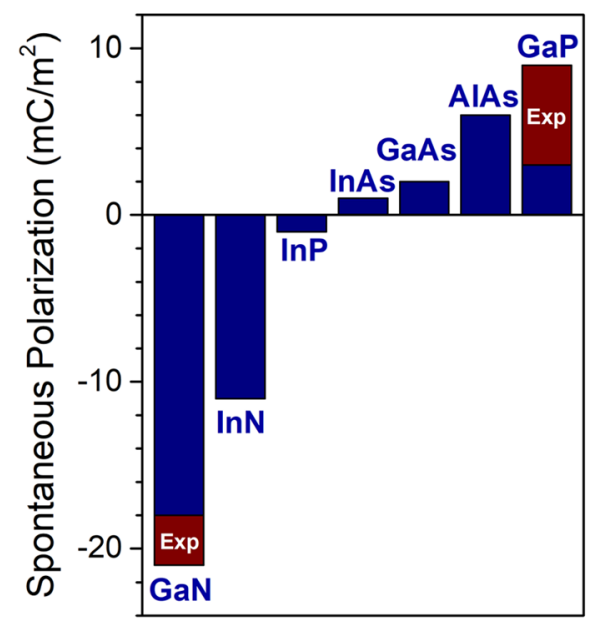

C

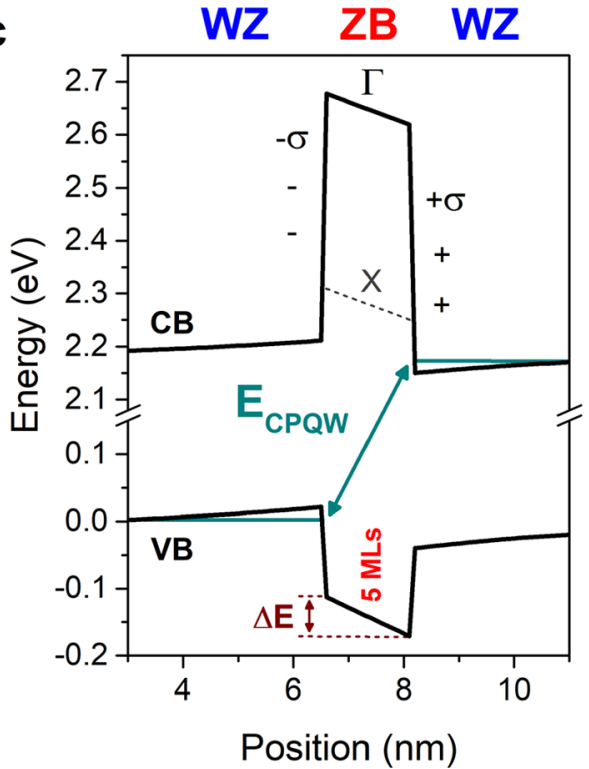

b

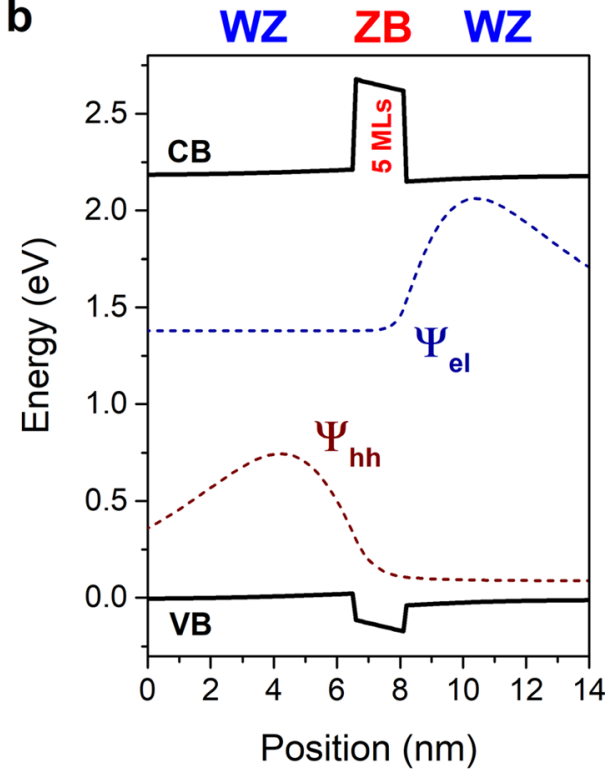

d $\quad 5$ MLs 6 MLs 7 MLs

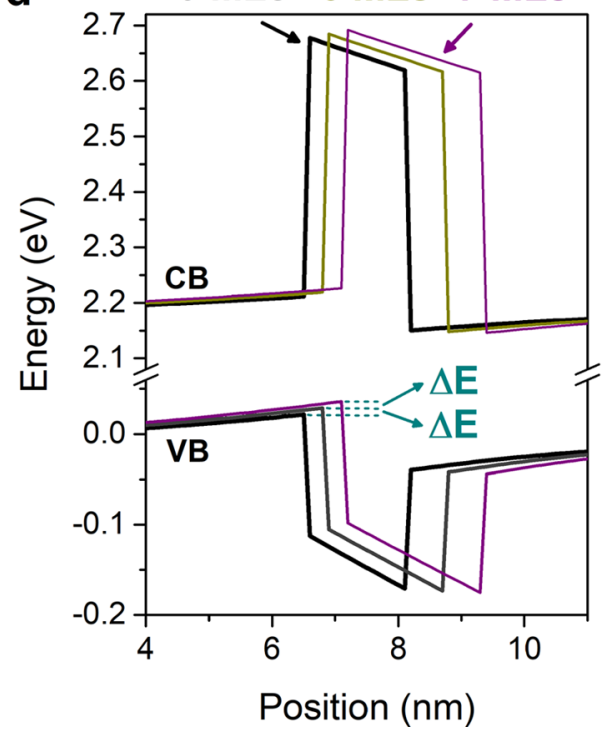

Figure 1. (a) Plot of the spontaneous polarization fields in III-V semiconductors. The dark blue areas are the values predicted from DFT, while the dark red areas are the values determined from experimental measurements. (b) Calculated band diagram for a CPQW in GaP with 5 ZB MLs using 1D Schrödinger-Poisson simulations. The dashed lines indicate the normalized wave functions for electrons (dark blue) and holes (dark red). Curves are shifted to allow easier comparison. (c) Enlarged view of panel b showing a capacitor-like charge configuration where the sheet of polarization charges and the related QCSE are sketched. (d) Digital tuning of the CPQW transition energy for increasing ZB thicknesses of 5, 6, and 7 MLs. By adding (removing) one monolayer, the resulting transition will be decreased (increased) by an amount of $\Delta E$.

microcrystals and nanowires made of nitride-based semiconductors. $^{22,25,26}$ In our WZ/ZB/WZ structures, the lengths $L_{\mathrm{ZB}}$ of the $\mathrm{ZB}$ segments are much shorter than the lateral dimensions, resulting essentially in a simple parallel plate capacitor. Thus, the polarization sheet charge density $\sigma$ can be estimated as ${ }^{22,25}$

$$
\sigma=\left|P_{\mathrm{SP}}\right|=\frac{\Delta V \varepsilon \varepsilon_{0}}{L_{\mathrm{ZB}}}
$$

where $\Delta V$ is the potential difference across the plate capacitor, $\varepsilon$ is the dielectric constant of the material, and $\varepsilon_{0}$ is the permittivity of free space. The energy shift $\Delta E$ for changing the thickness of the capacitor with a single $\mathrm{ZB}$ monolayer is $\Delta E=\Delta V / q$, where $q$ is the elementary charge. This relation results in a discrete red- shift (blue-shift) $\Delta E$ of the emission energy upon the addition (removal) of a single ZB monolayer.

Here, we demonstrate digital tuning of the GaP CPQW emission energy across a spectral range of $75 \mathrm{meV}$ by varying the thickness of the ZB segment. The correlation between photoluminescence measurements and band structure simulations shows that the strong polarization field in $\mathrm{GaP}$ induces carrier confinement in the WZ section of the CPQW. The presence of atomically sharp interfaces results in emission line widths well below conventional III-V QW heterostructures. Therefore, the possibility to tailor the growth of WZ/ZB homojunctions in nanowires and thereby to digitally tune their emission energies provides a new tool in the design of solid-state 
quantum emitters in small diameter nanowires featuring true QD emission.

In the WZ unit cell, the bond length between the Ga and $\mathrm{P}$ atoms along the $\langle 0001\rangle$ axis ( $c$-axis) is determined as a fraction $u$ of the lattice parameter $c$, where $u$ is called the internal cell parameter. In an ideal WZ unit cell with tetrahedral bonds, $u=3$ / $8=0.375$, and the resulting spontaneous polarization $P_{S P}$ is equal to zero, which corresponds to the situation in the $\mathrm{ZB}$ unit cell. In WZ GaP nanowires, the measured value for the internal parameter is $u=0.37385 \pm 0.00017,{ }^{27}$ resulting in the prediction of a fairly large polarization $P_{\mathrm{SP}}^{\mathrm{GaP}}=9 \mathrm{mC} / \mathrm{m}^{2}$ along the $c$-axis. For comparison, calculations by density functional theory predict a value of $P_{\mathrm{SP}}^{\mathrm{GaP}}=3 \mathrm{mC} / \mathrm{m}^{2}$, ${ }^{21}$ which is still higher than in other III$\mathrm{V}$ semiconductors like GaAs, InAs, or InP, as summarized in Figure $1 a^{21,27,28}$ Similarly, a significant spontaneous polarization with opposite sign $(u>3 / 8)$ is known from nitride-based materials. $^{21,22}$ As discussed above, the interface charges at the $\mathrm{WZ} / \mathrm{ZB} / \mathrm{WZ}$ junctions can be treated as a parallel plate capacitor to derive the polarization field responsible for the change in emission energy per monolayer $(\mathrm{ML}){ }^{22} \mathrm{~A}$ more precise estimation of the evolution of the emission energy with $\mathrm{ZB}$ thickness can be performed using one-dimensional (1D) Schrödinger-Poisson calculations for the CPQWs along the $c$ axis (see Supporting Information S1 for more details). ${ }^{29}$ We note that, as the exciton Bohr radius in $\mathrm{GaP}$ is smaller than $8 \mathrm{~nm}$, no radial confinement is present in the $100 \mathrm{~nm}$-thick nanowires. ${ }^{19} \mathrm{In}$ addition, the lattice mismatch between $\mathrm{WZ}$ and $\mathrm{ZB}$ structures is very small, and the piezoelectric field $P_{\mathrm{PZ}}$ is negligible compared to the spontaneous polarization field $P_{\mathrm{SP}} \cdot{ }^{30}$ The type-I band profile for the $\mathrm{WZ} / \mathrm{ZB} / \mathrm{WZ} \mathrm{GaP}$ structures calculated using the 1D Schrödinger-Poisson method is presented in Figure $1 \mathrm{~b}, \mathrm{c}$. The calculated electron and hole wave functions depicted in Figure $1 \mathrm{~b}$ show a small overlap due to the presence of the $\mathrm{ZB}$ barrier, which implies a longer carrier lifetime. The energy corresponding to this spatially indirect transition depends on the thickness of the ZB segment. Every addition of a ZB monolayer reduces the transition energy by an amount $\Delta E$, as shown in Figure 1d, enabling digital tuning of the $\mathrm{GaP} \mathrm{CPQW}$ emission lines.

For optical characterization of CPQWs, WZ GaP nanowires incorporating multiple defect-free $\mathrm{WZ} / \mathrm{ZB} / \mathrm{WZ}$ homojunctions with atomically sharp interfaces were investigated. ${ }^{6}$ A representative WZ GaP nanowire of $100 \mathrm{~nm}$ diameter with multiple $\mathrm{ZB}$ segments is shown in the transmission electron microscopy (TEM) image in Figure 2a, where ZB sections with different lengths are separated by $200 \mathrm{~nm}$ long WZ segments. When considering the whole statistical ensemble of nanowires used for optical studies, ZB segments with lengths typically ranging between 3 and 60 MLs were investigated. As an example, ZB segments intentionally grown with lengths of $12 \mathrm{MLs}$ and 33 MLs are shown in the high-resolution TEM (HRTEM) images in Figure 2b,c.

The optical emission of the CPQW is investigated using photoluminescence (PL) spectroscopy. ${ }^{19,30,31}$ For this study, a high density of nanowires is dispersed on $\mathrm{a} \mathrm{SiO}_{x} / \mathrm{Si}$ substrate, and the optical emission is collected while scanning across the nanowire ensemble. Three different PL spectra of GaP nanowires with CPQWs are shown in Figure 3a, together with one PL spectrum from a pure $\mathrm{WZ} \mathrm{GaP} \mathrm{nanowire.} \mathrm{The} \alpha$ and $\gamma$ peaks at 2.140 and $2.251 \mathrm{eV}$ are related to bound excitons in $\mathrm{WZ} \mathrm{GaP}{ }^{19}$ whereas the additional sharp emission lines appear in a spectral region below the $\gamma$-line. This spectral region does not show any
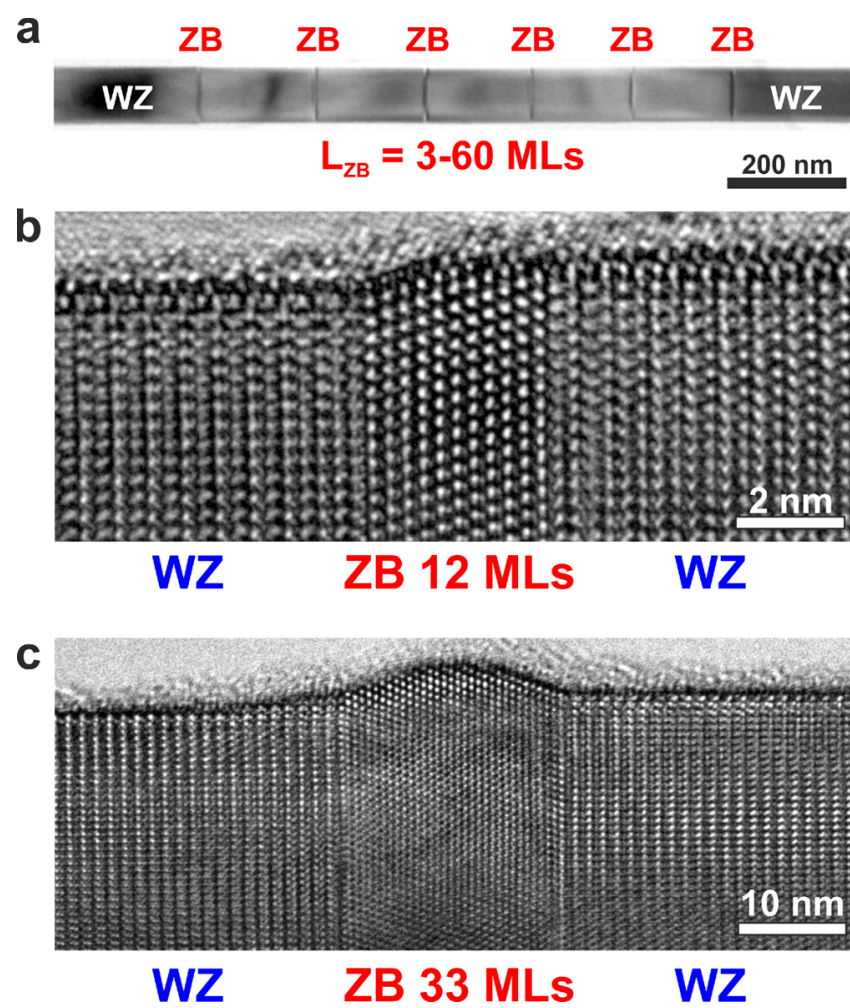

Figure 2. (a) TEM image of a WZ GaP nanowire with $100 \mathrm{~nm}$ diameter and multiple CPQWs of different ZB lengths separated by $200 \mathrm{~nm}$ long WZ segments. $(b, c)$ HRTEM images of CPQWs with ZB segments of 12 MLs (b) and 33 MLs (c) in length.

emission lines in pure WZ GaP nanowires, clearly demonstrating that these peaks originate from the embedded CPQW segments. The CPQW emission lines show an average full width at halfmaximum (fwhm) of $387 \pm 13 \mu \mathrm{eV}$ (see Figure $3 \mathrm{~b}$ ), reaching values as small as the setup resolution limit of about $\sim 200 \mu \mathrm{eV}$ (see Supporting Information Figure S2). This line width is remarkably sharp for a III-V semiconductor QW and is the direct consequence of the presence of atomically sharp interfaces. ${ }^{32}$ Once inhomogeneous broadening due to interface roughness is absent, the remaining broadening mechanism is due to charge fluctuations, ${ }^{33}$ either due to nonresonant excitation in the CPQW barrier or due to charged impurities,which are unintentionally incorporated within the NW or at the NW surface. As a confirmation that the emission lines originate from the $\mathrm{WZ}$ sections of the CPQW, we performed polarizationdependent PL measurements on a single NW, as shown in the inset of Figure $3 \mathrm{~b}$. The emission from the CPQW is polarized perpendicular to the long nanowire $c$-axis $\langle 0001\rangle$. This is in contrast to the isotropic behavior expected for carriers confined within a $\mathrm{ZB} \mathrm{GaP}$ segment. ${ }^{34,35}$ This observation strengthens the assumption of a type-I WZ/ZB junction with carrier confinement in the WZ segments, as predicted from band structure calculations. $^{12}$ A clear indication of the presence of different CPQWs in the PL spectra of Figure $3 \mathrm{a}$ is the regular spacing between multiple emission lines. To determine the emission energies of segments with a specific thickness in view of the variable emission energy, we performed a statistical analysis of the emission energy of hundreds of nanowires with CPQWs as summarized in the histogram in Figure 3c. Due to the occasional presence of the bound exciton $\beta$-line at $2.164 \mathrm{eV}$ in $\mathrm{WZ} \mathrm{GaP},{ }^{19}$ the investigated energy range has been limited to $2.168-2.246$ 
a

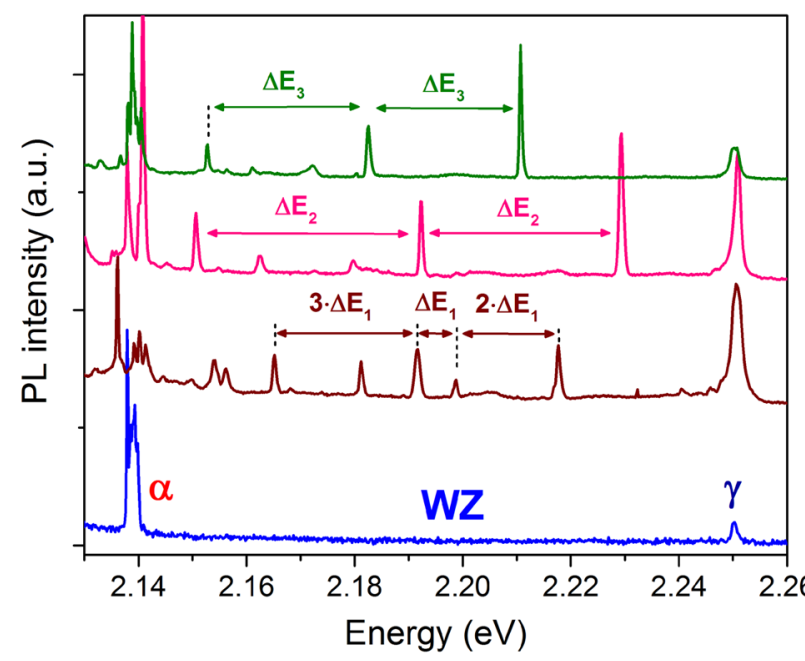

C

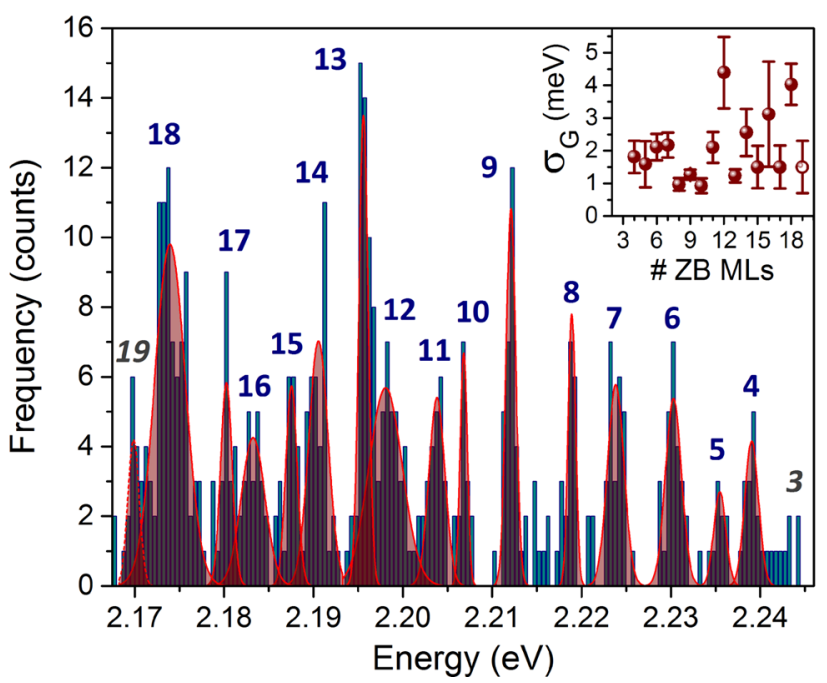

b

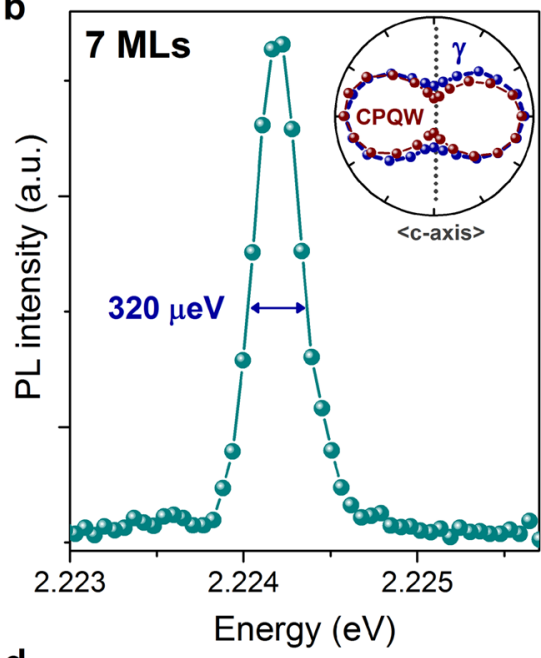

d

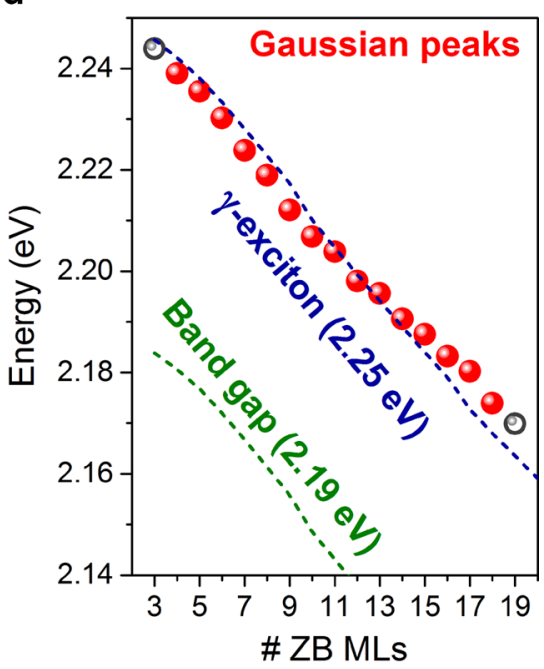

Figure 3. (a) PL spectra acquired for different CPQWs and compared with the emission from WZ GaP. (b) Enlarged view of a CPQW peak with a fwhm of $\sim 320 \mu \mathrm{eV}$. Inset: polarization-dependent PL measurement on a single NW with CPQWs. (c) Statistical analysis of the emission energy of hundreds of nanowires with CPQWs. Fifteen Gaussian peaks are used for fitting, with additional peaks at 2.170 and $2.244 \mathrm{eV}$, with low statistical count being visible. $\mathrm{A}$ bin size of $0.5 \mathrm{meV}$ is used for the histogram. Inset: width $\sigma_{\mathrm{G}}$ of the different Gaussian histogram peaks as a function of the ZB thickness. (d) Emission energy as a function of the number of ZB MLs (red spheres). The low statistical count peaks at 2.170 and $2.244 \mathrm{eV}$ are indicated with hollow grey spheres. The 1D Poisson simulations using the WZ band gap of $2.19 \mathrm{eV}$ (dashed dark green line) and $\gamma$-line bound exciton level at $2.25 \mathrm{eV}$ (blue dashed line) are shown.

eV. Strikingly, from the multi-Gaussian peak fit of the histogram in Figure 3c, we observe up to 15 clear peaks, with additional peaks at 2.170 and $2.244 \mathrm{eV}$ with low statistical counts. The presence of peaks in the histogram clearly shows that the CPQW emission lines are not random but instead exhibit a constant spacing of $\sim 5 \mathrm{meV}$ (see Figure 3d). This confirms that the position of the CPQW peaks is indeed governed by the respective number of $\mathrm{ZB}$ monolayers. Therefore, by tailoring the length of the $\mathrm{ZB}$ segments during growth, ${ }^{6} \mathrm{CPQW}$ emitting at the same energy can be designed with a high degree of control. Since the shortest $\mathrm{ZB}$ segment in a WZ wire has a length of 3 MLs, ${ }^{6}$ the first observable Gaussian peak should be associated with a ZB section of at least 3 MLs. The values for the standard deviation $\sigma_{G}$ of the Gaussian peaks are in the range of $1-5 \mathrm{meV}$ (inset Figure 3c). We tentatively explain the increased broadening of the histogram peak with increasing barrier thickness by a non-negligible number of charged impurities located within the $\mathrm{ZB}$ barrier at our background impurity concentration of $6 \times 10^{17} \mathrm{~cm}^{-3}$ (see below). Charged impurities will locally change the confinement potential resulting in fluctuations of the emission energy, as reported for stacking faults in $\mathrm{GaN}^{36}$ or InP QDs. ${ }^{37}$ We note that the standard deviation of $\sigma_{\mathrm{G}}=1-2 \mathrm{meV}$ for the shorter segments is comparable to state-of-the-art QD samples ${ }^{38}$ and probably can be further reduced by lowering the background impurity concentration.

The constant spacing of $\sim 5 \mathrm{meV} / \mathrm{ML}$ is proportional with the polarization charge minus the compensating free charges due to background doping, which tend to accumulate near the $\mathrm{ZB}$ interface. For this reason, the energy shift per monolayer was calculated using the 1D Schrödinger-Poisson calculations as shown by the dashed line in Figure $3 \mathrm{~d}$. An unintentional background doping level of $6 \times 10^{17} \mathrm{~cm}^{-3}$ as estimated from atom-probe tomography measurements on WZ GaP nanowires was considered in the model. ${ }^{19}$ At this doping level, the calculations reproduce the experimentally observed trend for a spontaneous polarization of $P_{\mathrm{SP}}=4.6 \mathrm{mC} / \mathrm{m}^{2}$ (see Supporting Information S1 for more details). For a doping level of $1 \times 10^{17}$ 

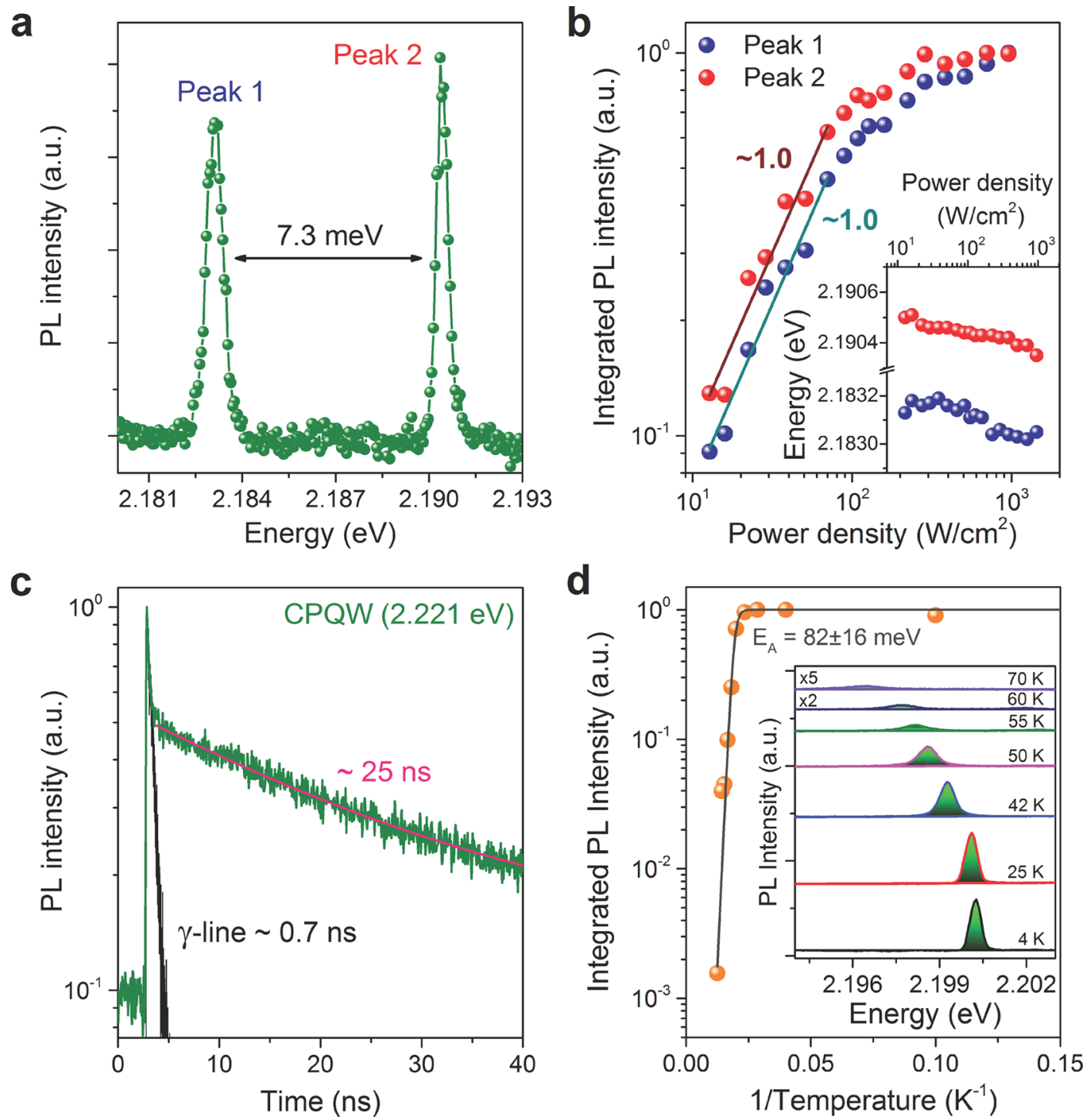

Figure 4. (a) PL spectra from two different QWs with a difference in thickness of $1 \mathrm{ZB}$ ML. (b) Integrated PL intensity as a function of the excitation power density for the peaks in panel a. Inset: peak energy as a function of the excitation power density. (c) Time-resolved PL measurements on a CPQW peak at $2.221 \mathrm{eV}$ compared with the time decay for the $\gamma$-exciton line. (d) Integrated PL intensity as a function of the reciprocal temperature for a CPQW at $2.220 \mathrm{eV}$. The fit is performed considering one active nonradiative recombination channel. Inset: PL spectra for increasing temperature.

$\left(1 \times 10^{18}\right) \mathrm{cm}^{-3}$, the experimental data would be reproduced for a polarization value of $2.8(5.5) \mathrm{mC} / \mathrm{m}^{2}$, which gives an estimate of the accuracy of the derived value. As the charge distribution due to doping is not taken into account, the plate capacitor approximation is not fully sufficient-in contrast to $\mathrm{GaN}$, where the spontaneous polarization is 5 times larger. ${ }^{22}$ A first calculation has been performed considering the WZ GaP bandgap of $2.19 \mathrm{eV}$, as shown by the green dashed line in Figure $3 \mathrm{~d} .{ }^{19}$ However, despite the agreement with the trend of the experimental data, a clear offset in energy is present. Therefore, we performed a second calculation considering the $\gamma$-line bound exciton level at $2.25 \mathrm{eV}$ for the $\mathrm{WZ}$ phase. ${ }^{19}$ In this case, the resulting agreement with the experimental energies shows that the CPQW emission is related to a very efficient recombination channel associated with the localized $\gamma$-transition in WZ GaP. For comparison, the value we find for the spontaneous polarization $P_{\mathrm{SP}}$ is slightly higher than the $3 \mathrm{mC} / \mathrm{m}^{2}$ predicted by DFT calculations, ${ }^{21}$ but a factor of 2 lower than the estimate from the internal cell parameter $u$ measured by X-ray diffraction. ${ }^{27}$
Power-dependent PL measurements were performed on emission lines at different energies to confirm that the emissions originate from different CPQWs. The two CPQW peaks in Figure $4 \mathrm{a}$ are separated by $7.3 \mathrm{meV}$, which differs from the expected shift of $\sim 5 \mathrm{meV}$ for the addition/removal of a ZB ML (see Figure $3 \mathrm{~d}$ ) possibly due to local fluctuations in doping concentrations. Looking at the excitation power dependence of the PL intensity as shown in Figure 4b, a linear scaling with slope $\sim 1.0$ is observed for both CPQW lines, followed by a saturation regime above $100 \mathrm{~W} / \mathrm{cm}^{2}$ Furthermore, the absence of a blueshift of the emission energy with increasing power (see inset Figure $4 b$ ) indicates that the number of photogenerated carriers is very small compared to the number of polarization charges, thus avoiding a blue shift due to compensation of the spontaneous polarization field by the photoinjected carriers. As a confirmation of the carrier separation in CPQWs, we performed time-resolved PL (TRPL) measurements, as shown in Figure 4c. The measured lifetime for different CPQW emission lines ranges between 20 and 30 ns. A clear correlation between increase in lifetime with increasing emission energy was 
not observed. These values are much larger than the lifetime of 0.5-1.0 ns observed in WZ GaP in the same energy range and of the $0.70 \mathrm{~ns}$ lifetime for the $\gamma$-line. ${ }^{19}$ Since the charge carriers are spatially separated and the transition is across a quantum barrier, the overlap of the electron and hole wave functions is reduced, which results in a decrease of the oscillator strength for the CPQW transition and hence in an increase of the radiative lifetime. However, we note that the presence of nonradiative recombination channels could affect the estimation of the lifetime from TRPL measurements, limiting our observations to a more qualitative picture. When the temperature of the system is increased, the emission from the CPQWs is quenched due to the thermal escape of holes out of the WZ confinement potential, as shown in Figure 4d. From fitting the temperature dependence of the integrated PL intensity of a CPQW at $2.200 \mathrm{eV}$ using a single nonradiative recombination channel, ${ }^{19}$ we find an activation energy $E_{\mathrm{A}}=82 \pm 16 \mathrm{meV}$. In $\mathrm{GaP}$, the $\mathrm{WZ} / \mathrm{ZB}$ valence band offset is $\sim 135 \mathrm{meV}$ (see Figure 1), ${ }^{12}$ and considering the presence of quantized levels that reduce the energy barrier, the good agreement with $E_{\mathrm{A}}$ confirms the confinement of both electrons and holes in the WZ segment of the CPQW.

In this work, we have provided experimental evidence for the presence of a spontaneous polarization in $\mathrm{WZ} \mathrm{GaP,} \mathrm{which} \mathrm{has} \mathrm{a}$ strong effect on the optical properties of crystal phase homojunctions in this material system. We demonstrated digital tuning of the emission energy associated with $\mathrm{WZ}-\mathrm{ZB}-\mathrm{WZ}$ crystal phase quantum well structures by tailoring the length of the $\mathrm{ZB}$ segments. This approach will allow to grow crystal phase quantum dots (CPQDs) by decreasing the NW diameter, which is a step toward the controlled fabrication of multiple CPQDs emitting identical photons. Identical single photon emitters are a key ingredient for the realization of entangled states in quantum information processing.

\section{ASSOCIATED CONTENT}

\section{S Supporting Information}

The Supporting Information is available free of charge on the ACS Publications website at DOI: 10.1021/acs.nanolett.7b02489.

Additional information on band structure calculations and optical characterization (PDF)

\section{AUTHOR INFORMATION}

\section{Corresponding Author}

*E-mail: j.e.m.haverkort@tue.nl.

\section{ORCID}

S. Assali: 0000-0002-3919-9112

J. Lähnemann: 0000-0003-4072-2369

K. D. Jöns: 0000-0002-5814-7510

L. Gagliano: 0000-0003-4214-8639

E. P. A. M. Bakkers: 0000-0002-8264-6862

J. E. M. Haverkort: 0000-0003-3051-673X

\section{Present Addresses}

${ }^{\perp}$ Department of Engineering Physics, Polytechnique Montréal, C. P. 6079, Succ. Centre-Ville, Montréal, Québec H3C 3A7, Canada.

\#Applied Physics Department, Royal Institute of Technology, Albanova University Centre, Roslagstullsbacken 21, 10691 Stockholm, Sweden.

${ }^{\nabla}$ Department of Photonics Engineering, Technical University of Denmark, 2800 Kongens Lyngby, Denmark.

\section{Notes}

The authors declare no competing financial interest.

\section{ACKNOWLEDGMENTS}

Maaike Bouwes Bavinck is acknowledged for the support in the PL measurements. This work was supported by the Dutch Organization for Scientific Research (NWO-VICI 700.10.441) and the Foundation for Fundamental Research on Matter (FOM). Solliance is acknowledged for funding the TEM facility.

\section{REFERENCES}

(1) Högele, A.; Seidl, S.; Kroner, M.; Karrai, K.; Warburton, R. J.; Gerardot, B. D.; Petroff, P. M. Phys. Rev. Lett. 2004, 93 (21), 217401.

(2) Seidl, S.; Kroner, M.; Högele, A.; Karrai, K.; Warburton, R. J.; Badolato, A.; Petroff, P. M. Appl. Phys. Lett. 2006, 88 (20), 203113.

(3) Bennett, A. J.; Pooley, M. A.; Stevenson, R. M.; Ward, M. B.; Patel, R. B.; de la Giroday, A. B.; Sköld, N.; Farrer, I.; Nicoll, C. A.; Ritchie, D. A.; Shields, A. J. Nat. Phys. 2010, 6 (12), 947-950.

(4) Rastelli, A.; Ding, F.; Plumhof, J. D.; Kumar, S.; Trotta, R.; Deneke, C.; Malachias, A.; Atkinson, P.; Zallo, E.; Zander, T.; Herklotz, A.; Singh, R.; Křápek, V.; Schröter, J. R.; Kiravittaya, S.; Benyoucef, M.; Hafenbrak, R.; Jöns, K. D.; Thurmer, D. J.; Grimm, D.; Bester, G.; Dörr, K.; Michler, P.; Schmidt, O. G. Phys. Status Solidi B 2012, 249 (4), 687-696.

(5) Aharonovich, I.; Englund, D.; Toth, M. Nat. Photonics 2016, 10 (10), 631-641.

(6) Assali, S.; Gagliano, L.; Oliveira, D. S.; Verheijen, M. A.; Feiner, L.F.; Plissard, S. R.; Bakkers, E. P. A. M. Nano Lett. 2015, 15 (12), 80628069.

(7) Lehmann, S.; Jacobsson, D.; Dick, K. A. Nanotechnology 2015, 26 (30), 301001.

(8) Loitsch, B.; Winnerl, J.; Grimaldi, G.; Wierzbowski, J.; Rudolph, D.; Morkötter, S.; Döblinger, M.; Abstreiter, G.; Koblmüller, G.; Finley, J. J. Nano Lett. 2015, 15 (11), 7544-7551.

(9) Caroff, P.; Dick, K. A.; Johansson, J.; Messing, M. E.; Deppert, K.; Samuelson, L. Nat. Nanotechnol. 2009, 4 (1), 50-55.

(10) Vainorius, N.; Lehmann, S.; Jacobsson, D.; Samuelson, L.; Dick, K. A.; Pistol, M.-E. Nano Lett. 2015, 15 (4), 2652-2656.

(11) Akopian, N.; Patriarche, G.; Liu, L.; Zwiller, V. Nano Lett. 2010, 10 (4), 1198-1201.

(12) Belabbes, A.; Panse, C.; Furthmüller, J.; Bechstedt, F. Phys. Rev. B: Condens. Matter Mater. Phys. 2012, 86 (7), 75208.

(13) Dick, K. A.; Thelander, C.; Samuelson, L.; Caroff, P. Nano Lett. 2010, 10 (9), 3494-3499.

(14) Bolinsson, J.; Caroff, P.; Mandl, B.; Dick, K. A. Nanotechnology 2011, 22 (26), 265606.

(15) Bouwes Bavinck, M.; Jöns, K. D.; Zieliński, M.; Patriarche, G.; Harmand, J. C.; Akopian, N.; Zwiller, V. Nano Lett. 2016, 16 (2), 10811085.

(16) Dean, P.; Thomas, D. Phys. Rev. 1966, 150 (2), 690-703.

(17) Morgan, T. N. Phys. Rev. Lett. 1968, 21 (12), 819-823.

(18) Bechstedt, F.; Belabbes, A. J. Phys.: Condens. Matter 2013, 25 (27), 273201.

(19) Assali, S.; Greil, J.; Zardo, I.; Belabbes, A.; de Moor, M. W. A.; Koelling, S.; Koenraad, P. M.; Bechstedt, F.; Bakkers, E. P. A. M.; Haverkort, J. E. M. J. Appl. Phys. 2016, 120 (4), 44304.

(20) Standing, A.; Assali, S.; Gao, L.; Verheijen, M. A.; van Dam, D.; Cui, Y.; Notten, P. H. L.; Haverkort, J. E. M.; Bakkers, E. P. A. M. Nat. Commun. 2015, 6, 7824.

(21) Belabbes, A.; Furthmüller, J.; Bechstedt, F. Phys. Rev. B: Condens. Matter Mater. Phys. 2013, 87 (3), 35305.

(22) Lähnemann, J.; Brandt, O.; Jahn, U.; Pfüller, C.; Roder, C.; Dogan, P.; Grosse, F.; Belabbes, A.; Bechstedt, F.; Trampert, A.; Geelhaar, L. Phys. Rev. B: Condens. Matter Mater. Phys. 2012, 86 (8), 081302.

(23) Fiorentini, V.; Bernardini, F.; Della Sala, F.; Di Carlo, A.; Lugli, P. Phys. Rev. B: Condens. Matter Mater. Phys. 1999, 60 (12), 8849-8858.

(24) Miller, D. A. B.; Chemla, D. S.; Damen, T. C.; Gossard, A. C.; Wiegmann, W.; Wood, T. H.; Burrus, C. A. Phys. Rev. Lett. 1984, 53 (22), 2173-2176. 
(25) Lähnemann, J.; Jahn, U.; Brandt, O.; Flissikowski, T.; Dogan, P.; Grahn, H. T. J. Phys. D: Appl. Phys. 2014, 47, 423001.

(26) Hille, P.; Müßener, J.; Becker, P.; De La Mata, M.; Rosemann, N.; Magén, C.; Arbiol, J.; Teubert, J.; Chatterjee, S.; Schörmann, J.; Eickhoff, M. Appl. Phys. Lett. 2014, 104, 102104.

(27) Kriegner, D.; Assali, S.; Belabbes, A.; Etzelstorfer, T.; Holý, V.; Schülli, T.; Bechstedt, F.; Bakkers, E. P. A. M.; Bauer, G.; Stangl, J. Phys. Rev. B: Condens. Matter Mater. Phys. 2013, 88 (11), 115315.

(28) Bauer, B.; Hubmann, J.; Lohr, M.; Reiger, E.; Bougeard, D.; Zweck, J. Appl. Phys. Lett. 2014, 104 (21), 211902.

(29) Snider, G. 1D Poisson freeware; University of Notre Dame. http://www.nd.edu/ gsnider/ (accessed May 1, 2015).

(30) Greil, J.; Assali, S.; Isono, Y.; Belabbes, A.; Bechstedt, F.; Valega Mackenzie, F. O.; Silov, A. Y.; Bakkers, E. P. A. M.; Haverkort, J. E. M. Nano Lett. 2016, 16 (6), 3703-3709.

(31) Assali, S.; Zardo, I.; Plissard, S.; Kriegner, D.; Verheijen, M. A.; Bauer, G.; Meijerink, A.; Belabbes, A.; Bechstedt, F.; Haverkort, J. E. M.; Bakkers, E. P. A. M. Nano Lett. 2013, 13 (4), 1559-1563.

(32) Grundmann, M. The Physics of Semiconductors; Springer, 2006.

(33) Lodahl, P.; Mahmoodian, S.; Stobbe, S. Rev. Mod. Phys. 2015, 87 (2), 347-400.

(34) Mishra, A.; Titova, L. V.; Hoang, T. B.; Jackson, H. E.; Smith, L. M.; Yarrison-Rice, J. M.; Kim, Y.; Joyce, H. J.; Gao, Q.; Tan, H. H.; Jagadish, C. Appl. Phys. Lett. 2007, 91 (26), 263104.

(35) Wilhelm, C.; Larrue, A.; Dai, X.; Migas, D.; Soci, C. Nanoscale 2012, 4 (5), 1446.

(36) Corfdir, P.; Lefebvre, P.; Ristić, J.; Ganière, J.-D.; DeveaudPlédran, B. Phys. Rev. B: Condens. Matter Mater. Phys. 2009, 80 (15), 153309.

(37) Reimer, M. E.; Bulgarini, G.; Fognini, A.; Heeres, R. W.; Witek, B. J.; Versteegh, M. A. M.; Rubino, A.; Braun, T.; Kamp, M.; Höfling, S.; Dalacu, D.; Lapointe, J.; Poole, P. J.; Zwiller, V. Phys. Rev. B: Condens. Matter Mater. Phys. 2016, 93 (19), 195316.

(38) Juska, G.; Dimastrodonato, V.; Mereni, L. O.; Gocalinska, A.; Pelucchi, E. Nat. Photonics 2013, 7, 527-531. 